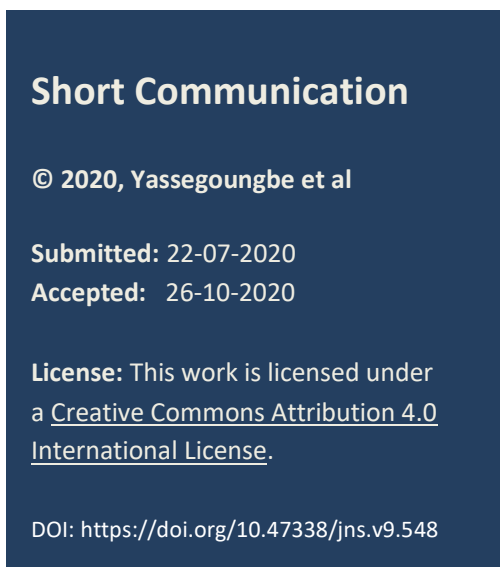

\title{
Gastrointestinal perforations in newborns with high mortality: A series of 18 cases
}

Monsoia Gildas Yassegoungbe, ${ }^{1}$ Beaudelaire Romulus Assan, ${ }^{1}$ Amoussou Sedjro Clotaire Romeo Houegban, ${ }^{1}$ Djifid Morel Seto, ${ }^{* 2}$ Codjo Serge Metchihoungbe, ${ }^{1}$ Eudes Ulrich Elvis Mahougnon Goudjo, ${ }^{1}$ Houenoukpo Koco, ${ }^{1}$ Sourou Bruno Noukpozounkou, ${ }^{3}$ Aldrico Pautin Covi, ${ }^{1}$ Michel Armand Fiogbe, ${ }^{1}$

1 Department of Pediatric Surgery, National Teaching Hospital Hubert Koutoukou Maga, Cotonou, Benin 2 Department of General Surgery, District Hospital of Allada, Allada, Benin

3 Department of Pediatric Surgery, Mother and Child Teaching Hospital Lagune, Cotonou, Benin

Correspondence*: Djifd Morel Seto, Department of General Surgery, District Hospital of Allada, Allada, Benin E-mail: seto.morel@gmail.com

\author{
KEYWORDS \\ Gastrointestinal perforation, \\ NEC, \\ Mortality, \\ Prematurity, \\ Neonate
}

\begin{abstract}
Gastrointestinal perforations (GIP) in newborns are serious neonatal conditions that have significant morbidity and mortality, especially in resource constrained setups. This study was done to describe our experience of GIP in neonates and identify the factors leading to high mortality in our setup. We collected 18 cases with GIP in neonates. The average age was 5.7 days (range, 1-7 days). The sex ratio was 0.64 . The average time to treatment was 3.3 days (range, 1-10 days). The etiology of GIP was perforated necrotizing enterocolitis (13 patients), spontaneous gastric perforation ( 2 patients), perforated ileal atresia (1 patient), ileal perforation complicating a strangulated inguinoscrotal hernia ( 1 patient), and peritonitis after colostomy (1 patient). Mortality was $77.8 \%$ ( $n=14)$, among which 9 newborns $(64.3 \%)$ died preoperatively. Prematurity, management delay, and lack of a neonatal intensive care unit were the main poor prognostic factors. Mortality from GIP is still high in our context due to several factors, especially prematurity, management delay, and lack of a neonatal intensive care unit.
\end{abstract}

Gastrointestinal perforations (GIP) in newborns are serious conditions and categorized as spontaneous perforations (without obvious etiology) and perforations caused by an underlying pathology (secondary).[1-3] Among secondary perforations, necrotizing enterocolitis (NEC) is the most common cause of GIP.[4,5] Irrespective of the cause, a delay in the diagnosis and management leads to poor prognosis.[4] In sub-Saharan Africa, factors such as the lack of means and neonatal resuscitation can worsen the prognosis. Herein, we describe data of our patients with neonatal GIP and review the main problems related to the management of GIP in newborns in our setup.

This case series was carried out from December 2016 to December 2018, focusing on the newborns treated for GIP. The following data were analyzed: age at diagnosis, sex, birth weight, the status of prematurity, time to admission after the development of symptoms, clinical presentation, time to surgical management, surgical treatment, complications, and outcome. We categorized premature babies in our series as small prematurity (range $34-36$ weeks +6 days), medium prematurity (range $32-33$ weeks +6 days), and high prematurity (below 32 weeks). Data analysis was performed using SPSS statistics 25.0.

Of the 18 neonates with GIP, 7 were males and 11 females (M:F 1:1.57). The mean age at diagnosis was 5 days (range, 1-7 days). The average weight was 2355 g (range, 1120-3500g). Low birth weight $(<2500 \mathrm{~g})$ was noted in $10(55.6 \%)$ newborns and prematurity in $9(50 \%)$ (1 small premature, 6 medium premature, and 2 high premature). Clinically, the average time to admission was 3.3 days (range, 1-10 days). All the patients presented with abdominal distension, accompanied by vomiting in $94.4 \%(n=17)$. X-ray abdomen was performed in all patients which revealed pneumoperitoneum in 17 cases $(94.4 \%)$. The etiologies of GIP were; necrotizing enterocolitis (NEC) (13 cases, $72.2 \%$ ), spontaneous gastric perforation (2 cases, $11.1 \%$ ), perforated ileal atresia (1 case, 5.6\%), ileal perforation due to strangulated inguinoscrotal hernia ( 1 case, $5.6 \%$ ), peritonitis after colostomy for anorectal malformation (1 case, 5.6\%). Note that $69.2 \%(9 / 13)$ of newborns with enterocolitis were premature. Nine newborns were operated including 3 
cases of NEC in which peritoneal drainage was performed, 2 cases of NEC treated by immediate resection-anastomosis, 2 cases of gastric perforations treated by primary repair with omentoplasty, 1 case of strangulated hernia complicated by ileal perforation treated by immediate resection-anastomosis, and 1 case of perforated ileal atresia treated by resectionanastomosis. The average time to surgical management was 3 days (range, 2-5 days).

Mortality was $77.8 \%$ (14 newborns). Nine neonates died before any kind of surgery including 8 cases of NEC, and 1 case of peritonitis after colostomy. The causes of death were respiratory distress in 5 cases $(5$ cases of NEC) and septicemia in 4 cases ( 3 cases of $\mathrm{NEC}$, and 1 case of peritonitis after colostomy). In the postoperative cohort, 5 neonates died including 4 cases of NEC, and 1 case of perforated ileal atresia. The cause of death in this cohort was overwhelming sepsis. Lack of parenteral nutrition and the absence of a postoperative infant ventilator were the co-morbid factors found in our series. Several factors influenced the mortality of gastrointestinal perforations in newborns such as NEC, surgery performed beyond 72 hours, ileal perforation, prematurity, low birth weight, and the absence of neonatal resuscitation (parenteral nutrition, infant ventilator) (Table 1).

Table 1: Factors influencing the outcome of neonates with GIP

\begin{tabular}{|c|c|c|c|}
\hline & Total & Alive & Dead \\
\hline \multicolumn{4}{|c|}{ Diagnosis } \\
\hline NEC & 13 & 2 & 11 \\
\hline Others & 5 & 3 & 2 \\
\hline \multicolumn{4}{|c|}{ Delay of surgical treatment } \\
\hline$<72 \mathrm{~h}$ & 6 & 4 & 2 \\
\hline$\geq 72 \mathrm{~h}$ & 12 & 0 & 12 \\
\hline \multicolumn{4}{|c|}{ Site of perforation } \\
\hline Ilium & 13 & 3 & 10 \\
\hline Colon & 1 & 0 & 1 \\
\hline Stomach & 2 & 2 & 0 \\
\hline Multiple & 2 & 0 & 2 \\
\hline \multicolumn{4}{|c|}{ Gestational age } \\
\hline$<37 \mathrm{SA}$ & 9 & 2 & 7 \\
\hline$\geq 37 \mathrm{SA}$ & 9 & 4 & 5 \\
\hline \multicolumn{4}{|c|}{ Birth weight } \\
\hline$<2500$ & 11 & 1 & 10 \\
\hline$\geq 2500$ & 7 & 5 & 2 \\
\hline \multicolumn{4}{|c|}{ Suboptimal Neonatal resuscitation* } \\
\hline Yes & 14 & 0 & 10 \\
\hline No & 4 & 4 & 0 \\
\hline
\end{tabular}

In our study, NEC represents $72.2 \%$ of the causes of GIP, and nearly $70 \%$ of cases were premature. NEC is frequent in our series because of maternal-fetal infections, which are unrecognized due to the poor monitoring of pregnancies in our context. In $90 \%$ of cases, it occurs in premature infants.[6] This usual association of NEC with prematurity, which is often a contributing factor, makes this pathology serious and fatal, especially in our precarious context. In fact, in high premature newborns (<32 weeks gestational age), the onset often occurs during the fourth week after birth. In children born closer to term, onset usually occurs within the first week after birth.[3] Intestinal perforation occurs in about $20 \%$ of babies who develop NEC and is often multiple due to the fragility and immaturity of the intestine.[1] In addition, the lower the birth weight of a newborn, the higher the risk of developing NEC, low birth weight also being a predisposing factor alongside prematurity. In our series, 10 of the 11 cases with birth weight $<2500 \mathrm{~g}$ died.

We also identified 2 spontaneous gastric perforations, which account in literature for 10 to $16 \%$ of neonatal GIP, as also observed in our series, but the causes of these perforations were not elucidated except for fragility of the gastric wall.[5,7] The age of onset is variable between 2 and 7 days with a peak on the third day.[2,6] Dupont et al. indeed found a maximum gastric hyperacidity at day 3 of life, increasing the risk of perforation.[6] Other risk factors have been identified in the literature, particularly fetal distress, 
prematurity, low birth weight, amniotic infection, cesarean section, and hypoplasia of the gastric wall.[4,6-8] In our patients, in addition to fetal distress, gastric hypoplasia suspected during surgery was confirmed by pathological examinations.

Other common causes of secondary perforations include meconium ileus, meconium plug syndrome, birth defects obstructing the gastrointestinal tract, volvulus, and hernia.[1,2,4] Intestinal atresia is a rare cause of GIP, as was the case in our series. The perforation occurs in intestinal atresia if it is diagnosed or treated late as found in our context. $[1,2,4,9]$

Peritoneal drainage is a temporary measure to stabilize hemodynamically unstable newborns, especially premature and low birth weight babies.[1013] In our series, peritoneal drainage was given in cases with significant abdominal distension and respiratory compromise. This surgical technique allowed us to stabilize 3 cases of perforated necrotizing enterocolitis; but it was temporary because due to the lack of adequate neonatal resuscitation, these children did not survive until their condition was completely cured. Since its first description by Ein et al., several studies documented the successful management of GIP in some patients.[12,14] In fact, peritoneal drainage must be followed by good resuscitation, $[9,14,17]$ which is not the case in developing countries like ours.

Primary repair of the perforation is feasible in cases of GIP who present early. It is often difficult to achieve in

\section{REFERENCES}

1. Kuremu RT, Hadley GP, Wiersma R. Gastrointestinal tract perforation in neonates. Tropical Doctor. 2007; 37:1-3.

2. Ekwunife OH, Ugwu J, Modekwe V, Osuigwe AN. Gastrointestinal perforation in neonates: Aetiology and risk factors. J Neonatal Surg. 2013; 2:30.

3. Rich BS, Dolgin SE. Necrotizing enterocolitis. Eur J Pediatr Surg Rep. 2013; 1:30-1.

4. Asabe K, Oka Y, Kai HI, Shirakusa T. Neonatal gastrointestinal perforation. Turk J Pediatr. 2009; 51:264-70.

5. Naji A, Elkarout Y, Benjiba N. Spontaneous neonatal gastric perforation: about a case. Pan Afr Med J. 2015; 21:1-6.

6. Dupont P, Trentesaux A, Guillemin M, Petit T, JeannePasquier C, Guillois B. Idiopathic gastric perforations of the newborn in two cases. Gastroenterol Clin Biol. 2003; 27: $1160-2$.

7. Rakoto-Ratsimba HN, Rakotoarisoa B, Samison LH, Belalahy V, Ranaivozanany A. Spontaneous gastric perforation in a neonate: a case report. Archives de Pediatrie. 2004; 11:1067-9. cases of NEC because of extensive involvement or multiple perforations. Moreover, the inadequate neonatal resuscitation in our context is not suitable for the postoperative management of primary repair/anastomosis (lack of parenteral nutrition).

Neonatal GIP mortality is always high, ranging from 40 to $70 \% .[2,4,7]$ Persistent high mortality despite advances in anesthesia and neonatal intensive care has been attributed to increased survival of extremely premature babies.[2,18] NEC, low birth weight, sepsis, delayed management, and the existence of multiple perforations are also other factors that influence mortality.[2,9,18,19] All these factors explain the still high mortality in our country. This is further compounded by the problems of anesthesia and neonatal resuscitation in developing countries with a lack of a neonatal resuscitation unit and pediatric anesthesiologists.[2,20]

In conclusion, gastrointestinal perforations were mostly seen in premature neonates with necrotizing enterocolitis. Their mortality is still very high in our context due to several factors including, prematurity, management delay, ileal and multiple perforations, and lack of a neonatal intensive care unit

\section{Acknowledgements: Nil}

Conflict of Interest: Authors have no conflict of interest.

Source of Support: $\mathrm{Ni}$

Consent to Publication: No clinical figure is used in this manuscript.

Author Contributions: Author(s) declared to fulfil authorship criteria as devised by ICMJE and approved the final version.

8. Eicher C, Seitz G, Bevot A, Moll M, Goelz R, Arand J. Surgical management of extremely low birth weight infants with neonatal bowel perforation: a single-center experience and a review of the literature. Neonatol. 2012; 101:285-92.

9. Jawed AJ, Al-Rabie A, Hadi A, Al-Sowailem A, Al-Rawaf A, Abu-Touk B. Spontaneous neonatal gastric perforation. Pediatr Surg Int. 2002; 18:396-9.

10. Cass D, Brandt M, Patel DL, Nuchtern JG, Minifee PK, Wesson D. Peritoneal drainage as definitive treatment for newborns with isolated bowel perforation. J Pediatr Surg. 2000; 35:1531-6.

11. Ein SH, Marshall DG, Grivan D. Peritoneal drainage under local anesthesia for necrotizing enterocolitis. J Pediatr Surg. 1977; 12: 963-7.

12. Khan RA, Mahajan JK, Rao KLN. Spontaneous intestinal perforation in neonates: Is surgery always indicated? Afr J Paediatr Surg. 2011; 8:249-51.

13. Lacusso C, Boscarelli A, Fusaro F, Bagolan P, Morini F. Pathogenetic and prognostic factors for neonatal gastric perforation: Personal experience and systematic review of the literature. Frontiers in Pediatrics. 2018; 6:1-6.

14. Rees CM, Eaton S, Kiely EM, Wade AM, McHugh K, Pierro A. Peritoneal drainage or laparotomy for neonatal 
bowel perforation? A randomized controlled trial. Ann Surg. 2008; 248: 44-51.

15. Osifo OD, Ovueni ME. The prevalence, patterns, and causes of deaths of surgical neonates at two African referral pediatric surgical centers. Ann Pediatr Surg. 2009; 5:194-9.

16. Singh M, Owen A, Gull, S, Morabito A, Bianchi A. Surgery for intestinal perforation in preterm neonates: anastomosis vs stoma. J Pediatr Surg. 2006; 41:725-9.

17. Govani DR, Patel RR, Patel RV, More B, Bhimni SD. Pneumo-omentocele - a sign of silent lethal neonatal posterior gastric perforation. Austin J Clin Case Rep. 2014; 1:1038.

18. Ehrlich PF, Sato TT, Short BL, Hartman GE. Outcome of perforated necrotizing enterocolitis in the very low-birth weight neonate may be independent of the type of surgical treatment. Am Surg. 2001; 67:752-6.

19. Tiwari C, Sandlas G, Jayaswal S, Shah H. Spontaneous intestinal perforation in neonates. J Neonatal Surg. $2015 ; 4: 14$

20. Ameh EA. Challenges of neonatal surgery in subsaharan Africa. Afr J Paediatr Surg. 2004; 1:43-8. 